\title{
Different Adaptations in AMPA Receptor Transmission in the Nucleus Accumbens after Short vs Long Access Cocaine Self-Administration Regimens
}

\author{
Anthony Purgianto' ${ }^{1,3}$, Andrew F Scheyer ${ }^{1,3}$, Jessica A Loweth', Kerstin A Ford ${ }^{1,2}$, Kuei Y Tseng*,2,4 and \\ Marina E Wolf*,, 4
}

'Department of Neuroscience, Rosalind Franklin University of Medicine and Science, The Chicago Medical School, North Chicago, IL, USA;

${ }^{2}$ Department of Cellular and Molecular Pharmacology, Rosalind Franklin University of Medicine and Science, The Chicago Medical School, North Chicago, IL, USA

\begin{abstract}
$\mathrm{Ca}^{2+}$-permeable AMPA receptors (CP-AMPARs) accumulate in the nucleus accumbens (NAc) after $\sim$ I month of withdrawal from a long-access cocaine self-administration regimen ( $6 \mathrm{~h} / \mathrm{d}, \mathrm{IOd})$. This is functionally significant because CP-AMPARs mediate the 'incubated' cue-induced cocaine craving produced by this regimen. Our present goal was to determine if other commonly employed cocaine selfadministration regimens also elicit CP-AMPAR accumulation. We compared four regimens, named according to whether sessions were short-access (ShA, $2 \mathrm{~h}$ ) or long-access (LgA, 6h) and the total number of sessions: LgA/IOd (already shown to elicit CP-AMPAR accumulation), ShAll Id, ShA/20-24d, and LgA/20-24d. In the latter regimens, rats began with 10 days of ShA and then entered a differential phase (10-14 days) in which ShA sessions either continued or switched to LgA. Controls self-administered saline. After $>40$ days of withdrawal, whole-cell patch-clamp recordings were performed in NAc core medium spiny neurons to assess the contribution of CP-AMPAR transmission, based on the magnitude of synaptic suppression elicited by bath application of the selective CP-AMPAR antagonist naspm $(100 \mu \mathrm{M})$. Naspm produced a non-significant $(\sim 10 \%)$ attenuation of electrically evoked local excitatory postsynaptic current in the saline and ShA groups. By contrast, a significant naspm-induced synaptic attenuation (25-30\%) was observed in both the LgA groups. Further analyses indicate that this emergence of CP-AMPAR transmission in the LgA groups is associated with increased baseline responsiveness of MSN to excitatory drive. Together with data on cocaine infusions in each group, our results show that CP-AMPAR accumulation and enhanced glutamate transmission is associated with longer sessions (6h), rather than the number of sessions or cocaine infusions.
\end{abstract}

Neuropsychopharmacology (2013) 38, 1789-1797; doi: I0.1038/npp.20 3.78; published online 8 May 2013

Keywords: addiction \& substance abuse; AMPA receptors; animal models; cocaine; self-administration; glutamate; nucleus accumbens

\section{INTRODUCTION}

Medium spiny neurons (MSN), the output neurons of the nucleus accumbens (NAc), are excited primarily by AMPAtype glutamate receptors (AMPARs). Studies in animal models have shown that AMPAR transmission in the NAc is required for cocaine seeking, suggesting AMPAR plasticity as a key control point for craving and relapse (Kalivas and

*Correspondence: Dr KY Tseng, Department of Cellular and Molecular Pharmacology, Rosalind Franklin University of Medicine and Science, 3333 Green Bay Road, North Chicago, IL 60064, USA. E-mail: kuei-yuan.tseng@rosalindfranklin.edu.

Dr ME Wolf, Department of Neuroscience, Rosalind Franklin University of Medicine and Science, 3333 Green Bay Road, North Chicago, IL 60064, USA, Tel: +847 578 8659, Fax: +847 578 85I5, E-mail: marina.wolf@rosalindfranklin.edu

${ }^{3}$ These authors contributed equally to this work.

${ }^{4}$ These authors contributed equally to this work.

Received 9 November 2012; revised 26 February 2013; accepted 26 February 2013; accepted article preview online I April 2013
Volkow, 2005; Wolf and Ferrario, 2010). Many different cocaine regimens have been used to study cocaine-induced adaptations in AMPAR transmission in the NAc. Broadly speaking, they can be classified as non-contingent administration (cocaine is delivered by the experimenter), short-access self-administration (ShA; multiple sessions of 1-2 h/day), or long-access self-administration (LgA; multiple sessions of $4-6 \mathrm{~h} /$ day). There is evidence that regimens classified in this manner lead to different adaptations in the NAc as well as different behavioral outcomes (Wolf, 2010). Given the centrality of AMPAR transmission to NAc output, it would be helpful to know whether and how basic properties of AMPAR transmission are altered after different types of regimens, particularly ShA regimens which have been most widely utilized.

One important way to classify AMPARs is based on whether or not the tetrameric AMPAR includes the GluA2 subunit. GluA2-containing AMPARs are $\mathrm{Ca}^{2+}$-impermeable (CI-AMPARs), whereas those lacking GluA2 are $\mathrm{Ca}^{2+}$-permeable (CP-AMPARs). CP-AMPARs show higher 
single channel conductance and exhibit inward rectification due to voltage-dependent block by endogenous polyamines (Isaac et al, 2007; Lee, 2012). In the NAc of drug-naive rats or rats treated with non-contingent (experimenter-administered) cocaine, CI-AMPARs account for the vast majority of excitatory synaptic transmission in the NAc (Boudreau et al, 2007; Kourrich et al, 2007; Conrad et al, 2008; Ghasemzadeh et al, 2009; Ferrario et al, 2010; McCutcheon et al, 2011b). By contrast, CP-AMPARs accumulate in the core and shell subregions of the adult rat NAc after $\sim 1$ month of withdrawal from a particular LgA regimen (6h/day for 10 days) that leads to withdrawal-dependent intensification ('incubation') of cue-induced cocaine craving (Conrad et al, 2008; Ferrario et al, 2011; McCutcheon et al, 2011a,b; Wolf and Tseng, 2012). Importantly, studies conducted after $\sim 6$ weeks of withdrawal from this regimen showed that intra-NAc core injection of the selective CP-AMPAR antagonist naspm blocked the expression of incubation of cue-induced cocaine craving (Conrad et al, 2008). Thus, CP-AMPARs, which account for $\sim 30 \%$ of evoked EPSC amplitude at this withdrawal time, mediate the expression of incubation after prolonged withdrawal (Conrad et al, 2008). CP-AMPARs also accumulate in the mouse NAc after a similar regimen in association with enhanced cocaine craving (Mameli et al, 2009). From the standpoint of synaptic transmission, the presence of CPAMPARs is expected to have important consequences, such as providing a route for $\mathrm{Ca}^{2+}$ entry other than NMDARs and thus altering the 'rules' for induction of synaptic plasticity (eg, Mameli et al, 2011).

The goal of the present study was to determine whether CP-AMPARs accumulate in the NAc after withdrawal from several cocaine self-administration regimens that have been widely used in recent years. This is important for two major reasons. First, as already noted above, it will help to clarify the state of AMPAR transmission in the NAc, a critical determinant of NAc output, in a broad range of published studies that have examined cocaine-induced adaptations in the NAc. Second, we are presently engaged in developing strategies to reduce CP-AMPAR transmission and thereby reduce cue-induced cocaine craving (McCutcheon et al, 2011b; Loweth et al, 2013). It is important to know the circumstances in which these strategies may have utility.

We compared four regimens and named them according to duration of each session (ShA, $2 \mathrm{~h} ; \mathrm{LgA}, 6 \mathrm{~h}$ ) and the total number of sessions. First, we used our standard incubation regimen ( $6 \mathrm{~h} /$ day for 10 days; Conrad et al, 2008), hereafter termed $\mathrm{LgA} / 10 \mathrm{~d}$. We also assessed a ShA regimen that has been widely used ( $2 \mathrm{~h} /$ day for 10-12 days; eg, Moussawi et al, 2009; Knackstedt et al, 2010; Wiggins et al, 2011; Trantham-Davidson et al, 2012), hereafter termed ShA/11d. Finally, we included classical ShA and LgA regimens (Ahmed and Koob, 1998; Ahmed and Koob, 1999; Ahmed, 2011), which lead to different behavioral and cellular adaptations as addressed in the Discussion. The last two groups begin with a pre-training phase $(\sim 10$ days of ShA sessions) that is designed to avoid confounds associated with differential initial learning in the groups; then the ShA group remains at $2 \mathrm{~h} /$ day for another 10-14 days, while the LgA group switches to $6 \mathrm{~h} /$ day for 10-14 days. These two groups are termed $\mathrm{ShA} / 20-24 \mathrm{~d}$ and $\mathrm{LgA} / 20-24 \mathrm{~d}$, respectively. After $>40$ days of withdrawal, a period sufficient to enable CP-AMPAR accumulation following our standard incubation regimen $(\mathrm{LgA} / 10 \mathrm{~d})$, we used patch-clamp recordings to determine the contribution of CP-AMPARs to excitatory synaptic transmission onto MSN of the NAc core. Comparing ShA and LgA groups enabled us to determine the effect of session duration on AMPAR plasticity. Furthermore, the inclusion of two ShA groups with different numbers of sessions (ShA/11d and ShA/2024d) and two LgA groups with different numbers of sessions ( $\mathrm{LgA} / 10 \mathrm{~d}$ and $\mathrm{LgA} / 20-24 \mathrm{~d}$ ) enabled us to determine whether the number of sessions also influences the nature of AMPAR plasticity.

\section{MATERIALS AND METHODS}

All experimental procedures were performed according to the USPHS Guide for Care and Use of Laboratory Animals and were approved by the Rosalind Franklin University of Medicine and Science Institutional Animal Care and Use Committee.

\section{Animals and Cocaine Self-Administration}

Our procedures for animal preparation and cocaine selfadministration have been described in detail previously (Conrad et al, 2008; Ferrario et al, 2011; McCutcheon et al, 2011a,b). Briefly, adult male Sprague-Dawley rats (250$275 \mathrm{~g}$ on arrival; Harlan, Indianapolis, IN) were housed singly in standard rat cages (lights on at 1900 hours, off at 0700 hours; food and water available ad libitum). After a week of acclimatization to the animal colony, rats were implanted with a jugular catheter under isoflurane anesthesia. Immediately after surgery and every day after surgery, the catheters were flushed with sterile saline solution to ensure patency of the catheter. After 5-7 days of recovery, rats began self-administration training in operant chambers (MED Associates, St Albans, VT) using different regimens described in the following section. All self-administration sessions were conducted during the dark cycle. Nose-poking in the inactive hole had no consequences, whereas nose-poking in the active hole delivered an infusion of saline or cocaine $(0.5 \mathrm{mg} / \mathrm{kg}$ in a $100 \mu \mathrm{l} / \mathrm{kg}$ volume over $3 \mathrm{~s}$ ), paired with a $20 \mathrm{~s}$ light cue inside the nosepoke hole. Catheter patency was verified by flushing with saline before and after each self-administration session.

\section{Cocaine Regimens}

Background on each cocaine regimen is provided in the Introduction and Discussion sections. Rats in the ShA/11d group self-administered cocaine for $2 \mathrm{~h} / \mathrm{d}$ for 11 days. Rats in the LgA/10d group self-administered cocaine for $6 \mathrm{~h} /$ day for 10 days. LgA/20-24d and ShA/20-24d rats underwent an initial phase of training in which they self-administered cocaine for 10 days $(2 \mathrm{~h} /$ day $)$. These rats then entered a differential phase during which $\mathrm{LgA} / 20-24 \mathrm{~d}$ rats selfadministered cocaine for $6 \mathrm{~h} /$ day for $10-14$ additional days, while ShA/20-24d rats continued to self-administer cocaine for $2 \mathrm{~h}$ /day during that same period (approximately half of these rats had a 10-day differential phase, while the other half had a 14-day differential phase). Controls selfadministered saline according to either the ShA/20-24d or LgA/20-24d regimen. We did not use yoked controls 
because yoked delivery of cocaine is aversive and decreases subsequent motivation to obtain cocaine (Twining et al, 2009). After the last training session, rats were returned to their home cages for at least 40 days before electrophysiological recordings were performed.

\section{Electrophysiology}

All drugs and reagents were obtained from Sigma-Aldrich. Rats ( $459 \pm 20 \mathrm{~g}$ at the time of recording) were taken directly from their home cages and anesthetized with chloral hydrate (400-600 mg/kg, i.p.). Brains were rapidly removed and coronal slices $(300 \mu \mathrm{m})$ were cut using a vibrating microtome (VT1200S; Leica, Wetzlar, Germany) in ice-cold cutting solution (in mM: $122.5 \mathrm{NaCl}, 20$ glucose, $25 \mathrm{NaHCO}_{3}$, $2.5 \mathrm{KCl}, 0.5 \mathrm{CaCl}_{2}, 3 \mathrm{MgCl}_{2}, 1 \mathrm{NaH}_{2} \mathrm{PO}_{4}, 1$ ascorbic acid) constantly oxygenated with $95 \% \mathrm{O}_{2}-5 \% \mathrm{CO}_{2}$. Slices were then transferred to a warm $\left(32-34{ }^{\circ} \mathrm{C}\right)$ incubation chamber containing 95\% $\mathrm{O}_{2}-5 \% \quad \mathrm{CO}_{2}$ oxygenated artificial CSF (aCSF; in mM: $122.5 \mathrm{NaCl}, 20$ glucose, $25 \mathrm{NaHCO}_{3}, 2.5$ $\mathrm{KCl}, 0.5 \mathrm{CaCl}_{2}, 3 \mathrm{MgCl}_{2}, 1 \mathrm{NaH}_{2} \mathrm{PO}_{4}, 1$ ascorbic acid) for at least $1 \mathrm{~h}$ after slicing. For recordings, $\mathrm{CaCl}_{2}$ was increased to $2.5 \mathrm{mM}, \mathrm{MgCl}_{2}$ was reduced to $1 \mathrm{mM}$ and $0.1 \mathrm{mM}$ picrotoxin was added. All recordings were conducted at $32-34^{\circ} \mathrm{C}$. Patch pipettes (6-8 M 2 ) were pulled from $1.5 \mathrm{~mm}$ borosilicate glass capillaries (World Precision Instruments, Sarasota, FL) with a horizontal puller (P-97, Sutter Instruments, Novato, CA, USA) and filled with a potassium-based solution (in mM: $140 \mathrm{KCl}, 10 \mathrm{HEPES}, 2 \mathrm{MgCl}_{2}$, 5 NaATP, 0.6 NaGTP). MSNs in the NAc core were visualized using infrared (IR)-differential interference contrast video microscopy with a $40 \times$ water-immersion objective (Olympus BX51-WI, Olympus America, Center Valley, PA) connected to an IR-sensitive CCD camera and displayed on a monitor. Electrophysiologically, MSNs were identified by their characteristic slow membrane potential ramp to subthreshold somatic depolarizing current steps. Whole-cell patch-clamp recordings were conducted with a computercontrolled Axo-patch 200B amplifier, digitized with Digidata $1322 \mathrm{~A}$ at a sampling rate of $10 \mathrm{kHz}$, and acquired using pClamp 9.2 (Molecular Devices). The liquid junction potential was not corrected and electrode potentials were adjusted to zero before obtaining the whole-cell configuration. As previously reported (McCutcheon et al, 2011a,b), a bipolar tungsten-stimulating electrode placed $\sim 300 \mu \mathrm{m}$ from the recording site was used to elicit EPSCs $(0.2 \mathrm{~ms}$ pulse, every 20-30 s). The intensity of stimulation was chosen from the minimum amount of current required to elicit a synaptic response with $<15 \%$ variability in amplitude during the first $10 \mathrm{~min}$ of recording. Only neurons that continued to exhibit this synaptic response reliability during the subsequent $15 \mathrm{~min}$ of baseline recording were included in the analysis. Naspm was then bathapplied for at least $10 \mathrm{~min}$ to neurons that met this criterion, during which time EPSCs were monitored as described above. Analyses for input-output data were conducted in cells for which a stimulation titration was performed. Briefly, current intensity of the stimulating electrode was increased in $25 \mu \mathrm{A}$ steps starting at $50 \mu \mathrm{A}$. Following the identification of a synaptic response, stepwise titration was continued until a suitable and consistent EPSC was achieved, at least six steps $(150 \mu \mathrm{A})$ above rheobase.

\section{Statistical Analysis}

Data from the LgA and ShA groups were analyzed using two-way repeated-measures ANOVA with between-factor experimental groups and within-factor sessions of selfadministration. In all electrophysiological recordings, naspm-induced changes in EPSC amplitude relative to baseline values were assessed using paired $t$-tests. The magnitude of naspm sensitivity across the different experimental groups was further compared using one-way ANOVA. Data are expressed as mean \pm SEM. Effects were considered significant when $p<0.05$.

\section{RESULTS}

Rats were trained to self-administer cocaine or saline according to one of the four regimens described in Methods. All cocaine rats acquired self-administration as indicated by preference for the active hole over the inactive hole on all training days $\left({ }^{\star} p<0.05, t\right.$-tests comparing nosepokes in active and inactive holes for each group; data not shown). The number of infusions on each training day is shown in Figure 1a-c for cocaine groups that were trained with the ShA/11d regimen, the LgA/10d (incubation) regimen, and the ShA/20-24d or LgA/20-24d regimens, respectively. Saline controls, run in parallel with the $\mathrm{ShA} /$ $20-24 \mathrm{~d}$ and $\mathrm{LgA} / 20-24 \mathrm{~d}$ cocaine groups, are shown in Figure 1c. Infusions during each $2 \mathrm{~h}$ session were similar for cocaine rats in the ShA/11d and ShA/20-24d groups, as expected (compare panels a and $\mathbf{c}$ in Figure 1). During the differential phase, when the $\operatorname{LgA} / 20$-24d group switched to $6 \mathrm{~h} /$ day sessions while the ShA/20-24d group remained at $2 \mathrm{~h} /$ day, the number of infusions increased markedly in the LgA/20-24d group (Figure 1c) to levels significantly higher than the ShA/20-24d group $\left(\mathrm{F}_{(1,9)}=101.3, p<0.01\right)$. We limited the number of sessions for $\operatorname{LgA} / 20-24 \mathrm{~d}$ rats during their differential phase (10-14 sessions) so that they would be comparable with the number of $6 \mathrm{~h} /$ day sessions in the $\mathrm{Lg} / 10 \mathrm{~d}$ group (10 sessions). No significant escalation of intake was observed during the differential phase for the $\operatorname{LgA} / 20-24 \mathrm{~d}$ group $\left(\mathrm{F}_{(9,81)}=1.95, p=0.06\right)$, although there was a trend towards more infusions on the final training day (two-tailed, paired $t$-test, infusions on differential day $1 \mathrm{vs}$ differential day $10, p=0.08$ ). Similar trends were observed when only the first hour of self-administration was analyzed for the LgA/20-24d group (data not shown). The number of infusions per session was similar in the LgA/10d group and the LgA/20-24d group during its differential phase (compare panels $\mathbf{b}$ and $\mathbf{c}$ in Figure 1). The total number of infusions over all sessions for each experimental group is shown in Table 1. This is an accurate measure of cocaine intake because cocaine concentration in the syringe was the same for all the groups and delivery volume was adjusted for the weight of each rat.

After completion of the self-administration regimen, rats were returned to home cages for at least 40 days before electrophysiological analysis. This withdrawal period was selected because it is sufficient for CP-AMPAR accumulation to occur in the NAc following completion of our standard incubation regimen, here termed LgA/10d (Wolf and Tseng, 2012). After withdrawal, slices were prepared and whole-cell patch-clamp recordings were conducted in 

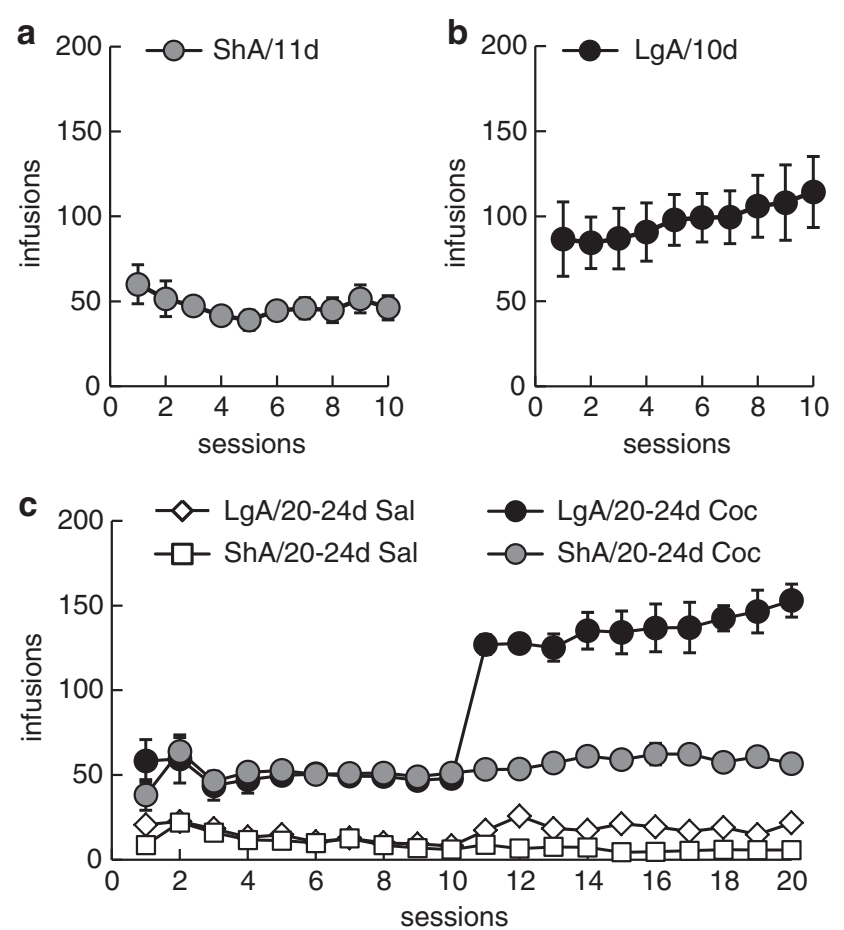

Figure I Rats were trained with four different self-administration regimens. Data depict mean \pm SEM number of infusions per session. For all rats receiving cocaine, the cocaine dose was $0.5 \mathrm{mg} / \mathrm{kg} / \mathrm{infusion}$. (a) In the ShAll Id paradigm ( $n=6$ cocaine), rats self-administered for $2 \mathrm{~h} /$ day over I I days. Infusions on day II are not shown but were nearly identical to day 10. (b) In the incubation paradigm $(n=7$ cocaine), rats self-administered for $6 \mathrm{~h} /$ day over 10 days. (c) In the LgA/20-24d ( $n=6$ cocaine, $n=7$ saline) and ShA/20-24d ( $n=7$ cocaine, $n=7$ saline) regimens, rats selfadministered for $2 \mathrm{~h} /$ day over 10 days during an initial phase, and then entered a differential phase in they were either switched to $6 \mathrm{~h} /$ day or maintained at $2 \mathrm{~h} /$ day (LgA/20-24d and ShA/20-24d, respectively). Although some rats self-administered for slightly longer than the number of days presented here (eg, about half of the LgA/20-24d rats had a differential phase lasting 14 days rather than 10 days), infusions are shown only for those training days when data for all rats in the group are available (eg, differential days $\mathrm{I}-10$ for the LgA/20-24d group).

MSN of the NAc core. In previous studies, we have shown that the LgA/10d regimen leads to CP-AMPAR accumulation in all nearly MSN sampled in the core (eg, McCutcheon et al, 2011b), which reduces concerns about whether different populations of MSN were sampled in the different experimental groups. To compare relative levels of $\mathrm{CP}$ AMPAR-mediated transmission in MSN synapses from each treatment group, we measured changes in the amplitude of electrically evoked EPSC following bath application of naspm $(100 \mu \mathrm{M}, 10 \mathrm{~min})$. Saline controls from the ShA/20$24 \mathrm{~d}$ and $\operatorname{LgA} / 20-24 \mathrm{~d}$ groups were combined because they did not differ. As expected from our previous studies (Conrad et al, 2008; Ferrario et al, 2011; McCutcheon et al, 2011a,b), naspm produced only a modest, non-significant reduction of the evoked EPSC amplitude in saline controls $(10.1 \pm 0.05 \%, p=0.37 ; n=7$ cells $/ 5$ rats; Figure $2 \mathrm{a})$. This appears to reflect a small population of CP-AMPARs based on experiments in which the effect of bath application of vehicle (aCSF alone) was examined. Over the same 10-min time-period, the rundown determined in this manner
Table I Total Number of Infusions Over All Self-Administration (SA) Sessions

SA regimen

Infusions (mean \pm SEM)

ShA/20-24d Sal

$173 \pm 23$

LgA/20-24d Sal

$325 \pm 52$

ShAll Id Coc

$47 \mid \pm 43$

ShA/20-24d Coc

$1088 \pm 58$

LgA/20-24d Coc

$1806 \pm 125$

LgA/IOd Coc

$973 \pm|6|$

(5.9 $\pm 0.03 \%, p=0.17 ; n=6$ cells $/ 6$ rats) was somewhat smaller than the percentage of reduction produced by naspm in the saline group (Figure 2f), although the difference was not statistically significant. Similar to results in saline controls, naspm produced a non-significant reduction of EPSC amplitude in slices from rats that selfadministered cocaine under ShA conditions $(\mathrm{ShA} / 11 \mathrm{~d}$ rats: $13.4 \pm 0.04 \%, p=0.66 ; n=5$ cells/ 4 rats; ShA/20-24d rats: $12.1 \pm 0.05 \%, p=0.43, n=7$ cells $/ 6$ rats; Figure $2 b-c)$. By contrast, after prolonged withdrawal from the LgA/20-24d regimen, naspm produced a significant reduction of the evoked EPSC amplitude $(23.8 \pm 0.05, p<0.05 ; n=8$ cells $/ 5$ rats; Figure 2d) that was comparable with that observed in the LgA/10d group $(28.8 \pm 0.04 \%, p<0.05 ; n=10$ cells/7 rats; Figure 2e). Thus, as summarized in Figure 2f, CPAMPAR-mediated synaptic transmission in the NAc core increased after $\operatorname{LgA}$ regimens but not ShA regimens.

We next determined whether the accumulation of CP-AMPARs is associated with an enhanced baseline glutamatergic transmission at NAc core MSN synapses. For this purpose, we assessed the magnitude of EPSC amplitude increase in response to synaptic stimulation as a function of current input above rheobase intensity. Here, rheobase is defined as the minimal synaptic stimulation current required to elicit an EPSC. For this analysis, the $\mathrm{LgA} / 10 \mathrm{~d}$ and $\mathrm{LgA} / 20-24 \mathrm{~d}$ groups were combined as they showed a similar pattern of response across a range of stimulation intensities above rheobase (group $\times$ intensity interaction, $\mathrm{F}_{(2,27)}=0.1, p=0.91$, two-way ANOVA). Similarly, data from both the ShA groups were pooled as the pattern of the evoked response was indistinguishable from each other (group $\times$ intensity interaction, $\mathrm{F}_{(2,24)}=0.02$, $p=0.98$, two-way ANOVA). Overall, we found that MSNs recorded from the LgA groups ( $n=11$ cells $/ 7$ rats) exhibited an increased responsiveness to synaptic stimulation when compared with the ShA groups $(n=10$ cells/7 rats) and saline controls $(n=7$ cells/4 rats; main group effect $\mathrm{F}_{(2,75)}=8.87, p<0.001$, two-way ANOVA; Figure 3). From the three ranges of current intensity tested, a marked increase in EPSC amplitude response was observed at 50 and $100 \mu \mathrm{A}$ above rheobase in the LgA groups. By contrast, the pattern of EPSC response obtained from the ShA groups was indistinguishable from that recorded in saline controls. Together, these results indicate that baseline glutamatergic synaptic transmission onto NAc MSNs becomes strengthened only following $\operatorname{LgA}$ regimens, concurrently with the accumulation of CP-AMPARs. 
a

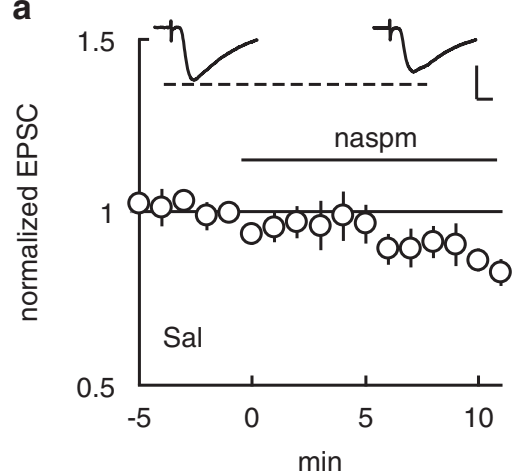

d

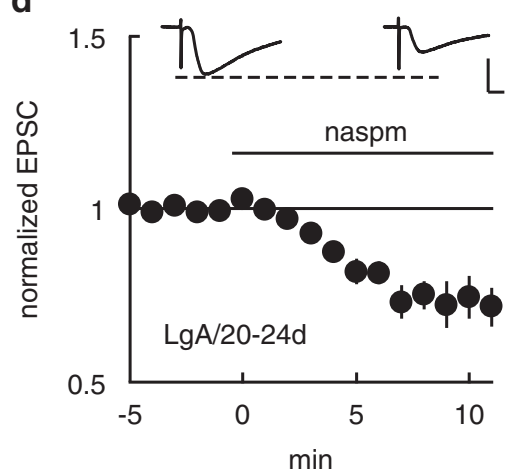

b

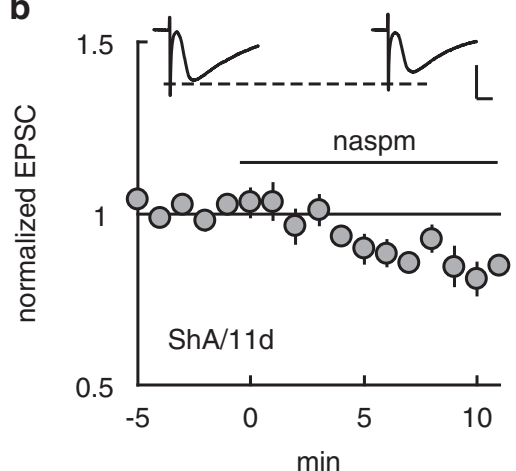

e

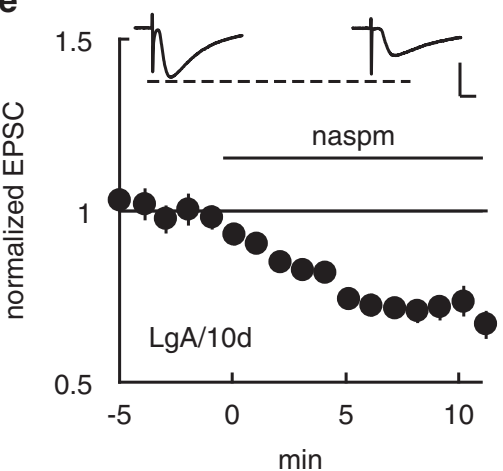

C

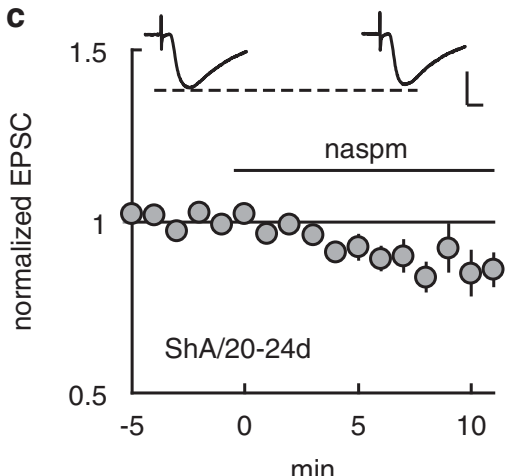

f

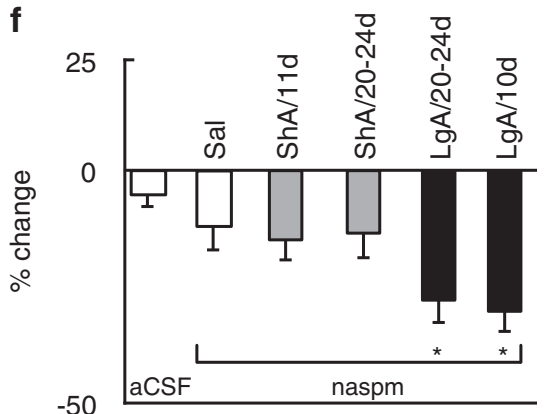

Figure 2 Naspm significantly decreases nucleus accumbens (NAc) excitatory synaptic transmission in LgA/I0d (incubation) and LgA/20-24d paradigms only, indicating a significant contribution of CP-AMPARs to excitatory synaptic transmission after LgA but not ShA cocaine self-administration. Rats selfadministered cocaine or saline as shown in Figure I. Recordings of NAc MSN were performed after 40-79 days of withdrawal in home cages, with each experimental group sampled at both shorter and longer withdrawal times within this range. (a) Saline (Sal) controls, $n=7$ cells ( 5 rats). Saline controls from ShA and LgA/20-24d groups were combined because they did not differ. (b) ShAlI Id cocaine rats, $n=5$ cells (4 rats). (c) ShA/20-24d cocaine rats, $n=7$ cells $(6$ rats). (d) LgA/20-24d cocaine rats, $n=8$ cells ( 5 rats). (e) LgA/IOd (incubation) cocaine rats, $n=10$ cells (7 rats). ( $f$ Summary of percentage of reduction in evoked EPSC amplitude after naspm in the experimental groups shown in panels a-e, as well as a control group in which bath application of vehicle (aCSF without naspm) was performed at $t=0$ min (aCSF group). ${ }^{*} p<0.05$ vs baseline (t-tests), and vs aCSF, Sal, ShA/I Id, ShA/20-24d groups (Fisher's LSD post-hoc test after significant one-way ANOVA, main treatment effect $F(5,39)=7.23, P<0.00005$ ).

Taken together, our behavioral and electrophysiological data indicate that session duration, rather than the number of sessions, was the important variable in determining the nature of AMPAR plasticity, as the LgA/10d and ShA/11d groups underwent approximately the same number of self-administration sessions yet only the $\operatorname{LgA} / 10 \mathrm{~d}$ group showed CP-AMPAR accumulation (Figure 2). Nor did overall cocaine exposure, expressed as the total number of cocaine infusions (Table 1), appear to be a critical variable. Thus, the number of infusions was very similar in the LgA/ $10 \mathrm{~d}$ and ShA/20-24d groups, yet only the LgA/10d group showed CP-AMPAR accumulation. Furthermore, the ShA/ 20-24d group had many more infusions than the ShA/11d group, yet neither group exhibited CP-AMPAR accumulation.

\section{DISCUSSION}

In the NAc of drug-naive rats, most AMPARs are GluA1A2or GluA2A3-containing, although a minority population of CP-AMPARs is detected with biochemical and electrophysiological techniques (Boudreau et al, 2007; Conrad et al, 2008; Reimers et al, 2011). In agreement with these previous results, we found that the selective CP-AMPAR antagonist naspm produced only a small, not statistically significant $\sim 10 \%$ reduction of the evoked EPSC amplitude in MSN of the NAc core recorded after $>40$ days of withdrawal from saline self-administration. Similar non-significant reductions were observed after ShA cocaine self-administration, indicating that the level of CP-AMPAR-mediated synaptic transmission in the NAc core following ShA regimens is indistinguishable from saline controls. By contrast, CP-AMPARs accounted for $\sim 25-30 \%$ of the evoked EPSC after $\operatorname{LgA}$ sessions (6h/day), regardless of whether these occur as part of a classical LgA/20-24d regimen or according to the regimen typically used to elicit incubation of cocaine craving (LgA/10d). Our results in the $\mathrm{LgA} / 10 \mathrm{~d}$ group replicate our previous reports that naspm decreases evoked EPSC amplitude by $\sim 25-30 \%$ after the same incubation regimen (Conrad et al, 2008; McCutcheon et al, 2011a). These results, together with data on total cocaine infusions in each group, indicate that CP-AMPAR accumulation depends on longer sessions ( $6 \mathrm{~h} v s \mathrm{~h}$ ), rather than the total number of sessions or the total amount of cocaine intake. Furthermore, our electrophysiological analysis demonstrated that CP-AMPAR accumulation was associated with an overall enhancement of MSN baseline responsiveness to excitatory stimulation. 


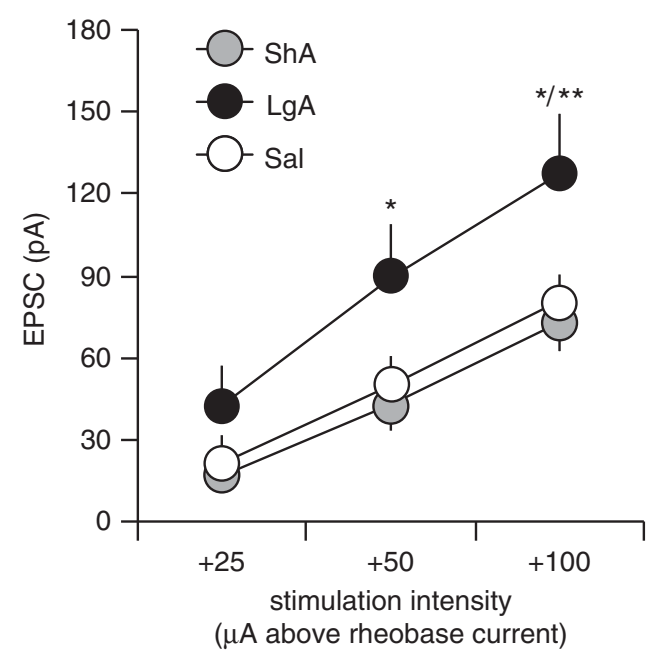

Figure 3 Baseline glutamatergic transmission at NAc excitatory synapses is enhanced after LgA regimens only. A two-way ANOVA revealed a significant main effect of group $\left(F_{(2,75)}=8.87, p<0.00 \mathrm{I}\right.$; LgA: $n=1$ I cells, 7 rats; ShA: $n=10$ cells, 7 rats; saline controls: $n=7$ cells, 4 rats). Note that the evoked EPSC amplitude was significantly enhanced in MSNs recorded from the LgA group as compared with the ShA group at $+50 \mu \mathrm{A}$ $(*)<0.05$, Fisher's LSD post-hoc test). Additionally, evoked EPSC amplitude was significantly enhanced after LgA cocaine self-administration as compared with ShA cocaine or saline self-administration at $+100 \mu \mathrm{A}$ ( ${ }^{*} p<0.05$ vs saline, *** $p<0.0$ I vs ShA, Fisher's LSD post-hoc test).

\section{AMPAR Plasticity after ShA Cocaine Self-Administration}

A major finding from our study is that ShA cocaine selfadministration, which has been very widely used to study cocaine-induced neuroadaptations in the NAc, does not lead to CP-AMPAR accumulation. However, our negative results do not preclude upregulation of CI-AMPARs after ShA cocaine self-administration. Indeed, a recent study found increased miniature EPSC amplitude and AMPA/NMDA ratios in the NAc shell after 3-4 weeks, but not 1-2 days, of abstinence from a ShA regimen (Ortinski et al, 2012). In light of our findings, it seems most likely that these were CI-AMPARs, although an important caveat is that our studies were performed in NAc core and at a significantly longer withdrawal time (40-79 days). However, there is an indirect support for AMPAR upregulation in the core after ShA cocaine self-administration. Thus, long-term potentiation (LTP) but not long-term depression (LTD) was impaired in the NAc core on withdrawal day 21 after a ShA regimen (Knackstedt et al, 2010). This could reflect occlusion of LTP by previous AMPAR upregulation, although there are many caveats associated with inferring AMPAR levels from changes in the ability to elicit LTP or LTD (Wolf, 2010; Wolf and Ferrario, 2010). Regardless of whether CI-AMPAR upregulation occurs in NAc core after 3-4 weeks abstinence, it may not be present at the later withdrawals examined here, based on the fact that inputoutput studies in Figure 3 did not reveal a difference between the ShA and saline groups. However, we cannot rule out the possibility of concurrent adaptations in the ShA groups that masked the consequences of CI-AMPAR upregulation.

Why does ShA cocaine self-administration fail to increase CP-AMPAR-mediated synaptic transmission? An obvious difference between ShA and LgA regimens is the total amount of cocaine intake (see Table 1). However, as noted in the introductory paragraph of the Discussion, this explanation is not supported by our data, as the $\operatorname{LgA} / 10 \mathrm{~d}$ and the ShA/20-24d groups had very similar numbers of cocaine infusions but only the former exhibited CP-AMPAR accumulation. Thus, in the present study, the duration of each self-administration session was the most important factor in determining the nature of AMPAR plasticity. Presumably, the longer activation of monoamine receptors that results from a longer duration session leads to more persistent activation of signaling pathways. There are many ways that this could be envisioned to 'flip a switch' (ie, CPAMPARs accumulate, drug seeking becomes compulsive, and so on); for example, persistent signaling pathway activation may be more likely to alter gene expression. It should be noted that the voluntary and learning aspects of cocaine self-administration are also important as we have shown that non-contingent cocaine exposure does not lead to CP-AMPAR accumulation in the NAc of adult rats (McCutcheon et al, 2011b).

\section{Functional Significance of CP-AMPAR Accumulation after LgA Regimens}

The functional role of CP-AMPARs has already been studied in the $\mathrm{LgA} / 10 \mathrm{~d}$ regimen that leads to incubation of cocaine craving. Incubation describes the progressive intensification of cue-induced craving during abstinence, measured as responding on the previously active lever or hole during an extinction test. Craving begins to incubate during the first week of withdrawal, increases progressively for $\sim 2$ months, and remains elevated even 6 months after the last selfadministration session (Lu et al, 2004; Pickens et al, 2011). By contrast, the rise in CP-AMPAR levels is first detected after 3-4 weeks of withdrawal (Wolf and Tseng, 2012), and the ability of naspm to block incubated cocaine craving was demonstrated on withdrawal day 45 (Conrad et al, 2008). Putting these findings together, other neuroadaptations must account for the initial phase of incubation, which is not surprising given that incubation involves multiple brain regions and cellular changes (Pickens et al, 2011). However, once CP-AMPARs are in place, their stimulation is required for the expression of incubation (Conrad et al, 2008). We have hypothesized that the synaptic incorporation of CP-AMPARs enhances the responsiveness of NAc neurons to glutamate inputs from cortical and limbic regions, due to their larger single channel conductance (Conrad et al, 2008; Wolf and Tseng, 2012). Support for this hypothesis is provided by the present input-output studies showing enhanced evoked EPSC amplitude in MSN from the LgA groups but not the ShA groups compared with saline controls. In addition, the $\mathrm{Ca}^{2+}$ permeability of CP-AMPARs is likely to qualitatively alter AMPAR signaling within the MSN. Thus, when cocaine-associated cues are presented after prolonged withdrawal from cocaine, and glutamate is released in the NAc, our results predict that the MSN will respond more robustly, leading to 'incubated' cue-induced cocaine seeking. The failure of ShA regimens to elicit CPAMPAR accumulation may explain why cue-induced seeking is greater in $\operatorname{LgA}$ than in ShA rats in tests conducted after 30-47 days of withdrawal (Lu et al, 2004; Ferrario et al, 2005). 
Is there any relationship of CP-AMPARs to other behavioral changes that follow LgA cocaine self-administration? It is well established that animals with LgA cocaine exposure demonstrate addiction-like behaviors not present in ShA animals, namely: (1) increased motivation for cocaine, (2) persistence of drug self-administration despite adverse consequences, and (3) augmented cocaine-, stress-, or cue-induced reinstatement following a period of extinction training (eg, Ahmed and Koob, 1998; Ahmed and Koob, 1999; Paterson and Markou, 2003; Deroche-Gamonet et al, 2004; Mantsch et al, 2004; Vanderschuren and Everitt, 2004; Ferrario et al, 2005; Kippin et al, 2006; Knackstedt and Kalivas, 2007; Mantsch et al, 2008; Ben-Shahar et al, 2008; reviewed by Ahmed, 2011). In most instances, these augmented behaviors were demonstrated within the first week or two after discontinuing LgA cocaine self-administration, a time period when CP-AMPAR levels have not yet increased (Wolf and Tseng, 2012). Thus, CP-AMPARs cannot be responsible for these augmented behaviors. Instead, it is more reasonable to propose that animals that develop these augmented behaviors subsequently exhibit elevated CP-AMPAR levels. Once this occurs, the CPAMPARs, which we have already demonstrated to underlie incubated cue-induced cocaine seeking (Conrad et al, 2008), may also contribute to other delayed behavioral changes that follow $\operatorname{LgA}$ cocaine self-administration. For example, after 30 days of abstinence, rats resume cocaine taking at an even higher level than occurred during their previous LgA training sessions (Ahmed and Koob, 1998). This could be related to increased activation of MSN due to the presence of CP-AMPARs, as discussed in the previous paragraph. It will be important to further characterize long-term behavioral consequences of LgA cocaine self-administration and the role of AMPAR plasticity in the NAc and other regions in such consequences.

\section{Absence of Significant Escalation in $\operatorname{LgA} / 20-24 \mathrm{~d}$ and LgA/11d Cocaine Groups}

Escalation to heavy drug use is a core diagnostic criterion for addiction; likewise, escalation of intake occurs in animals during LgA cocaine self-administration and is generally associated with other behaviors (see Discussion in the previous section) that model compulsive drug seeking and taking (Ahmed, 2011). We did not observe statistically significant escalation of cocaine intake in our LgA/20-24d group, which experienced 10-14 LgA sessions. In fact, while escalation is a reliable phenomenon under many conditions, there are other studies that have failed to detect it (Ahmed, 2011). Based on previous results (Mantsch et al, 2004; Kippin et al, 2006), it is likely that our use of Sprague-Dawley rats and a relatively low cocaine dose contributed to minimal escalation in our study. The timing of escalation also varies between studies. Sometimes, it is detected over the first 3-5 sessions (eg, Figure 1 of Ahmed and Koob, 1998; Figure 2 of Ben-Shahar et al, 2009), but it can require more sessions (19 sessions in Figure 3 of Ahmed and Koob, 1998). There are many procedural differences between these studies and ours; a particularly relevant difference may be that rats leverpressed for cocaine in the previous studies, whereas we trained rats to nose-poke for cocaine. Nose-pokes are easier to learn, which may work against the detection of escalation during early self-administration sessions.

We also failed to observe escalation in our $\operatorname{LgA} / 10 \mathrm{~d}$ group. We have run many identical groups over the past 5 years in our studies of incubation of cocaine craving (Conrad et al, 2008; Ferrario et al, 2011; McCutcheon et al, 2011a,b; additional unpublished studies). Sometimes, we observed escalation in the course of 10 sessions (eg, Ferrario et al, 2011). However, sometimes we and others have failed to observe escalation with this regimen (eg, Conrad et al, 2008; Knackstedt and Kalivas, 2007). Yet all incubation rats exhibit CP-AMPARs in the NAc core when analyzed after a month or so of withdrawal. Therefore, we conclude that LgA cocaine exposure can result in incubation of cocaine craving and synaptic accumulation of CP-AMPARs independent of whether we detect escalation over the course of 10 training sessions. Previous studies have shown that escalation can be dissociated from augmented cocaine- and cue-induced reinstatement after LgA cocaine self-administration (Kippin et al, 2006; Knackstedt and Kalivas, 2007), and compulsive drug seeking (eg, persistence despite aversive consequences) has been demonstrated after different types of LgA regimens where escalation was not tested (DerocheGamonet et al, 2004; Vanderschuren and Everitt, 2004). Together, these findings underscore the complexity of the relationships between different behavioral adaptations observed following different cocaine regimens.

\section{Therapeutic Implications}

In rats, the cocaine exposure required for the synaptic accumulation of CP-AMPARs in NAc synapses is not extreme ( $6 \mathrm{~h} /$ day $\times 10$ days and $\sim 1$ month of withdrawal). If a similar phenomenon occurs in humans, it may become important in situations where drug use is terminated for a month or two due to hospitalization or incarceration (Reichel and Bevins, 2009). After this period of abstinence, new CP-AMPARs could increase the likelihood of relapse by mediating intensified cue-induced cocaine craving. We are presently exploring mGluR1-based strategies for removing CP-AMPARs from NAc synapses and reducing cue-induced cocaine craving (McCutcheon et al, 2011a; Loweth et al, 2013).

Studies of incubation provide insight specifically into how to counteract cue-induced craving after prolonged withdrawal. Other regimens, including ShA regimens that do not lead to CP-AMPAR accumulation, provide insight into strategies for decreasing addictive behavior under different conditions. The most striking example concerns the drug $\mathrm{N}$-acetylcysteine, which reduces cocaine-related behaviors after non-contingent cocaine exposure and ShA cocaine self-administration and has shown promise in clinical trials (Mardikian et al, 2007; Amen et al, 2011; Olive et al, 2012).

\section{CONCLUSIONS}

A significant increase in CP-AMPAR levels was exhibited by MSN in the NAc core of rats after $>40$ days of withdrawal from LgA $(6 \mathrm{~h})$ cocaine self-administration sessions but not after ShA ( $2 \mathrm{~h})$ sessions. Based on earlier work showing that 3-4 weeks of withdrawal is required for CP-AMPAR accumulation, these high conductance AMPARs are likely 
to mediate delayed behavioral changes that follow LgA cocaine self-administration. Furthermore, the accumulation of CP-AMPARs (detected with naspm) was associated with enhanced baseline responsiveness of MSN to excitatory stimulation (detected by analysis of input-output relationships). By determining the experimental conditions that lead to CP-AMPAR accumulation, our results help evaluate the conditions under which CP-AMPAR-based therapeutic intervention might be valuable. Furthermore, our results enable conclusions to be drawn about the state of AMPAR transmission in the NAc in many studies in the literature that utilize the ShA and LgA models evaluated herein.

\section{ACKNOWLEDGEMENTS}

This research was supported by DA009621 to MEW and KYT, DA029099 to MEW, F32 DA030844 to JAL, and Rosalind Franklin University to KYT. We thank Dr Kelly Conrad and Dr Michela Marinelli for helpful comments on this manuscript.

\section{DISCLOSURE}

The authors declare no conflict of interest.

\section{REFERENCES}

Ahmed SH (2011). Escalation of drug use. In: Olmsted MC (eds). Animal Models of Drug Addiction, Neuromethods. Springer Science + Business Media, LLC2011 Vol 53(Part 2): pp 267-292.

Ahmed SH, Koob GF (1998). Transition from moderate to excessive drug intake: change in hedonic set point. Science 282: 298-300.

Ahmed SH, Koob GF (1999). Long-lasting increase in the set point for cocaine self-administration after escalation in rats. Psychopharmacology 146: 303-312.

Amen SL, Piacentine LB, Ahmad ME, Li S-J, Mantsch JR, Risinger $\mathrm{RC}$ et al (2011). Repeated N-acetylcysteine reduces cocaine seeking in rodents and craving in cocaine-dependent humans. Neuropsychopharmacology 36: 871-878.

Ben-Shahar O, Posthumus EJ, Waldroup SA, Ettenberg A (2008). Heightened drug-seeking motivation following extended daily access to self-administered cocaine. Prog Neuropsychopharmacol Biol Psychiatry 32: 863-869.

Ben-Shahar O, Obara I, Ary AW, Ma N, Mangiardi MA, Medina RL et al (2009). Extended daily access to cocaine results in distinct alterations in Homer $1 \mathrm{~b} / \mathrm{c}$ and NMDA receptor subunit expression within the medial prefrontal cortex. Synapse 63: 598-609.

Boudreau AC, Reimers JM, Milovanovic M, Wolf ME (2007). Cell surface AMPA receptors in the rat nucleus accumbens increase during cocaine withdrawal but internalize upon cocaine challenge in association with altered activation of mitogenactivated protein kinases. J Neurosci 27: 10621-10635.

Conrad KL, Tseng KY, Uejima JL, Reimers JM, Heng LJ, Shaham Y et al (2008). Formation of accumbens GluR2-lacking AMPA receptors mediates incubation of cocaine craving. Nature 454: 118-121.

Deroche-Gamonet V, Belin D, Piazza PV (2004). Evidence for addiction-like behavior in the rat. Science 305: 1014-1017.

Ferrario CR, Gorny G, Crombag HS, Li Y, Kolb B, Robinson TE (2005). Neural and behavioral plasticity associated with the transition from controlled to escalated cocaine use. Biol Psychiatry 58: 751-759.
Ferrario CR, Li X, Wang X, Reimers JM, Uejima JL, Wolf ME (2010). The role of glutamate receptor redistribution in locomotor sensitization to cocaine. Neuropsychopharmacology 35: 818-833.

Ferrario CR, Loweth JA, Milovanovic M, Ford KA, Galiñanes GL, Heng L-J et al (2011). Alterations in AMPA receptor subunits and TARPs in the rat nucleus accumbens related to the formation of $\mathrm{Ca}^{2+}$-permeable AMPA receptors during the incubation of cocaine craving. Neuropharmacology 61: 1141-1151.

Ghasemzadeh MB, Mueller C, Vasudevan P (2009). Behavioral sensitization to cocaine is associated with increased glutamate receptor trafficking to the postsynaptic density after extended withdrawal period. Neuroscience 159: 414-426.

Isaac JT, Ashby MC, McBain CJ (2007). The role of the GluR2 subunit in AMPA receptor function and synaptic plasticity. Neuron 54: 859-871.

Kalivas PW, Volkow ND (2005). The neural basis of addiction: a pathology of motivation and choice. Am J Psychiatry 162: 1403-1413.

Kippin TE, Fuchs RA, See RE (2006). Contributions of prolonged contingent and noncontingent cocaine exposure to enhanced reinstatement of cocaine seeking in rats. Psychopharmacology 187: 60-67.

Knackstedt LA, Kalivas PW (2007). Extended access to cocaine self-administration enhances drug-primed reinstatement but not behavioral sensitization. J Pharmacol Exp Ther 332: 1103-1109.

Knackstedt LA, Moussawi K, Lalumiere R, Schwend M, Klugmann M, Kalivas PW (2010). Extinction training after cocaine selfadministration induces glutamatergic plasticity to inhibit cocaine seeking. J Neurosci 30: 7984-7992.

Kourrich S, Rothwell PE, Klug JR, Thomas MJ (2007). Cocaine experience controls bidirectional synaptic plasticity in the nucleus accumbens. J Neurosci 27: 7921-7928.

Lee HK (2012). $\mathrm{Ca}^{2+}$-permeable AMPA receptors in homeostatic synaptic plasticity. Front Mol Neurosci 5: 17.

Loweth JA, Tseng KY, Wolf ME (2013). Using metabotropic glutamate receptors to modulate cocaine's synaptic and behavioral effects: mGluR1 finds a niche. Curr Opin Neurobiol e-pub ahead of print 2 February (doi:pii: S0959-4388(13)00025-1. 10.1016/j.conb.2013.01.009).

$\mathrm{Lu} \mathrm{L}$, Grimm JW, Hope BT, Shaham Y (2004). Incubation of cocaine craving after withdrawal: a review of preclinical data. Neuropharmacology 47: 214-226.

Mameli M, Halbout B, Creton C, Engblom D, Parkitna JR, Spanagel $\mathrm{R}$ et al (2009). Cocaine-evoked synaptic plasticity: persistence in the VTA triggers adaptations in the NAc. Nat Neurosci 12: 1036-1041.

Mameli M, Bellone C, Brown MT, Lüscher C (2011). Cocaine inverts rules for synaptic plasticity of glutamate transmission in the ventral tegmental area. Nat Neurosci 14: 414-416.

Mantsch JR, Yuferov V, Mathieu-Kia A-M, Ho A, Kreek MJ (2004). Effects of extended access to high versus low cocaine doses on self-administration, cocaine-induced reinstatement and brain mRNA levels in rats. Psychopharmacology 175: 26-36.

Mantsch JR, Baker DA, Francis DM, Katz ES, Hoks MA, Serge JP (2008). Stressor- and corticotropin releasing factor-induced reinstatement and active stress-related behavioral responses are augmented following long-access cocaine self-administration by rats. Psychopharmacology 195: 591-603.

Mardikian PN, LaRowe SD, Hedden S, Kalivas PW, Malcolm RJ (2007). An open-label trial of N-acetylcysteine for the treatment of cocaine dependence: a pilot study. Prog Neuropsychopharmacol Biol Psychiatry 31: 389-394.

McCutcheon JE, Loweth JA, Ford KA, Marinelli M, Wolf ME, Tseng KY (2011a). Group I mGluR activation reverses cocaine-induced accumulation of calcium-permeable AMPA receptors in nucleus accumbens synapses via a protein kinase C-dependent mechanism. J Neurosci 31: 14536-14541. 
McCutcheon JE, Wang X, Tseng KY, Wolf ME, Marinelli M (2011b). Calcium-permeable AMPA receptors are present in nucleus accumbens synapses after long withdrawal from cocaine self-administration but not experimenter-administered cocaine. J Neurosci 31: 5737-5743.

Moussawi K, Pacchioni A, Moran M, Olive MF, Gass JT, Lavin A et al (2009). N-Acetylcysteine reverses cocaine-induced metaplasticity. Nat Neurosci 12: 182-189.

Olive MF, Cleva RM, Kalivas PW, Malcolm RJ (2012). Glutamatergic medications for the treatment of drug and behavioral addictions. Pharmacol Biochem Behav 100: 801-810.

Ortinski PI, Vassoler FM, Carlson GC, Pierce RC (2012). Temporally dependent changes in cocaine-induced synaptic plasticity in the nucleus accumbens shell are reversed by D1-Like dopamine receptor stimulation. Neuropsychopharmacology 37: 1671-1682.

Paterson NE, Markou A (2003). Increased motivation for selfadministered cocaine after escalated cocaine intake. Neuroreport 14: 2229-2232.

Pickens CL, Airavaara M, Theberge F, Fanous S, Hope BT, Shaham Y (2011). Neurobiology of the incubation of drug craving. Trends Neurosci 34: 411-420.

Reichel CM, Bevins RA (2009). Forced abstinence model of relapse to study pharmacological treatments of substance use disorder. Curr Drug Abuse Rev 2: 184-194.
Reimers JM, Milovanovic M, Wolf ME (2011). Quantitative analysis of AMPA receptor subunit composition in addiction-related brain regions. Brain Res 1367: 223-233.

Trantham-Davidson H, LaLumiere RT, Reissner KJ, Kalivas PW, Knackstedt LA (2012). Ceftriaxone normalizes nucleus accumbens synaptic transmission, glutamate transport, and export following cocaine self-administration and extinction training. J Neurosci 32: 12406-12410.

Twining RC, Bolan M, Grigson PS (2009). Yoked delivery of cocaine is aversive and protects against the motivation for drug in rats. Behav Neurosci 123: 913-925.

Vanderschuren LJ, Everitt BJ (2004). Drug seeking becomes compulsive after prolonged cocaine self-administration. Science 305: 1017-1019.

Wiggins A, Smith RJ, Shen HW, Kalivas PW (2011). Integrins modulate relapse to cocaine-seeking. J Neurosci 31: 16177-16184.

Wolf ME (2010). The Bermuda triangle of cocaine-induced neuroadaptations. Trends Neurosci 33: 391-398.

Wolf ME, Ferrario CR (2010). AMPA receptor plasticity in the nucleus accumbens after repeated exposure to cocaine. Neurosci Biobehav Rev 35: 185-211.

Wolf ME, Tseng KY (2012). Calcium-permeable AMPA receptors in the VTA and nucleus accumbens after cocaine exposure: when, how and why? Front Mol Neurosci 5: 721. 